\title{
EFECTO HIPOGLICEMIANTE DEL EXTRACTO ACUOSO DE LAS HOJAS DE Smallanthus sonchifolius (YACÓN) EN PACIENTES CON DIABETES MELLITUS TIPO 2
}

\author{
The hypoglycemic effect of aqueous extract of leaves of Smallanthus sonchifolius (yacon) in \\ patients with Type 2 Diabetes Mellitus
}

Gloria C Gordillo, Luisa P Negrón, Teófila H Zúñiga, Eduardo Flores, Ronald Moreyra, César Fuertes, Gustavo A Guerra, Alfonso Apesteguía, Angélica M Quintana Facultad de Farmacia y Bioquímica, Universidad Nacional Mayor de San Marcos

\section{RESUMEN}

El presente estudio se realizó con el objetivo de evaluar el efecto hipoglicemiante de las hojas de Smallanthus sonchifolius (yacón), en la forma de infusión (extracto acuoso), administrada a pacientes con diabetes mellitus tipo 2. Previa firma de su consentimiento informado, participaron 206 personas de 30 a 70 años de edad, hombres y mujeres: 105 con diagnostico de diabetes mellitus tipo 2 y 101 aparentemente sanas, como grupo control. Los pacientes diabéticos tipo 2 fueron personas que acuden al Servicio Académico Asistencial de Análisis Clínicos (SAAAC), al Gabinete de Atención Farmacéutica (GAF) de la Facultad de Farmacia y Bioquímica, al Club de diabéticos tipo 2 del Hospital Cayetano Heredia, y al Centro de Salud (CERIT) Raúl Patrucco Puig. De los 105 diabéticos tipo 2 que recibían tratamiento farmacológico 46 estaban metabólicamente controlados $(\mathrm{Grupo} \mathrm{Al})$ y 59 no (Grupo Az). Los sujetos aparentemente sanos fueron divididos en dos grupos: 6o sujetos normales (Grupo B1) y 41 sujetos sin diabetes mellitus tipo 2 con mal control metabólico (Grupo B2). A los Grupos A2 y B2 se les proporcionó bolsas filtrantes de hojas de yacón de $1 \mathrm{~g}$ cada una, para ser tomadas en infusión tres veces al día por 9o días. El Grupo A2 continuó recibiendo glibenclamida. A todos se les determinó en ayunas la concentración de glucosa, hemoglobina glicosilada (HbAıc) y fructosamina, antes y después del tratamiento. Al final del tratamiento, se encontró que, la administración de la infusión de hojas de yacón disminuyó los valores de: glucosa en 42,7\%, hemoglobina glicosilada en 21,7 \% y fructosamina en $33,78 \%$, observándose una diferencia estadísticamente significativa con los valores basales. La eficacia de la combinación estudiada logra reducir las cifras de HbAic en 4,5\% a los 30 días y 21,7\% a los 90 días. En conclusión, la infusión de hojas de Smallanthus sonchifolius (yacón), administrada a pacientes con diabetes mellitus tipo 2, tiene un efecto protector beneficioso sobre el control glucémico.

Palabras clave: Smallanthus sonchifolius, yacón, diabetes mellitus tipo 2, hemoglobina glicosilada, fructosamina, glucosa.

\section{SUMMARY}

The present study was conducted to evaluate the hypoglycemic effect of Smallanthus sonchifflius (yacon) leaves, in the form of infusion (aqueous extract) administered to patients with type 2 diabetes mellitus. After signing informed consent, were included 206 people aged 30 to 70 years old, male and female: 105 diagnosed with type 2 diabetes mellitus and 101 apparently healthy control group. Type 2 diabetic patients were people who go to Scholar Healthcare of Clinical Analysis (SAAAC), Office of Pharmaceutical Care (GAF) of the Faculty of Pharmacy and Biochemistry, Club of type 2 diabetic patients of Cayetano Heredia Hospital and Patrucco Raul Puig Health Center (CERIT). From type 2 diabetic patients receiving drug treatment, there were 46 metabolically controlled patients and 59 not metabolically controlled ones. The latter group was given filter bags of yacon leaves of $1 \mathrm{~g}$ each, to be taken as an infusion three times a day for 90 days, plus glibenclamide. The apparently healthy subjects were divided into two groups, one ingested the yacon leaf tea and the other not. All were determined in fasting: glucose concentration, glycosylated hemoglobin (HbAic) and fructosamine before and after treatment. After treatment, it was found that administration of the leaf tea yacon values decreased by $42.7 \%$ glucose, glycosylated hemoglobin $21.7 \%$ and fructosamine $33,78 \%$, with a statistically significant difference with baseline values. The effectiveness of the studied combination is able to reduce HbAic by $4.5 \%$ at 30 days and $21.7 \%$ at 90 days. In conclusion, infusion of leaves Smallanthus sonchifolius (yacon) administered to patients with type 2 diabetes mellitus, has a beneficial protective effect on glycemic control.

Keywords: Smallanthus sonchifolius, Yacon, type 2 diabetes mellitus, glycated hemoglobin, fructosamine, glucose.

\section{INTRODUCCIÓN}

a diabetes mellitus tipo 2 es una enfermedad, endocrino metabólica crónica, caracterizada por un estado de deficiencia o resistencia a la insulina que conduce a un aumento de la glicemia, inicialmente con cambios en el metabolismo de carbohidratos y secundariamente de lípidos y proteínas ${ }^{(1-3)}$. Tiene gran alcance epidemiológico, requiere un tratamiento de por vida y un adecuado control metabólico. 
La OMS ha estimado que un gran porcentaje de la población mundial utiliza la medicina tradicional para satisfacer sus necesidades de atención primaria de salud y que gran parte de los tratamientos implica el uso de extractos de plantas o sus principios activos. Existe una tendencia a la utilización de productos naturales con fines farmacológicos, como complento al tratamiento de la diabetes mellitus tipo $2^{(3,4)}$.

Numerosas publicaciones hacen referencia a extractos obtenidos de plantas, eficaces en la reducción de la glicemia, que causan menos efectos secundarios y tienen un costo inferior al de los habituales agentes antidiabéticos ${ }^{(5-6)}$. Se ha demostrado que la mayoría de las plantas que se utilizan en la medicina tradicional para el tratamiento de la diabetes poseen componentes químicos biológicamente activos (alcaloides, glucósidos, cumarinas, flavonoides, terpenoides, sustancias fenólicas y otros) que pueden ser empleados como nuevos agentes hipoglicemiantes ${ }^{(6-7)}$.

El Smallanthus sonchifolius (yacón) es una planta perenne, herbácea, de la familia Asterácea, que se distribuye ampliamente en las regiones andinas (3). Diversos estudios han demostrado que sus hojas poseen diferentes efectos biológicos: inhibición de la migración de leucocitos polimorfonucleares, inmunomodulación, efectos antioxidante y citoprotector ${ }^{(8)}$. Trabajos previos reportan que la infusión de hojas de yacón reduce la glicemia y que probablemente los principios activos que contienen actúan estimulando la liberación de insulina y aumentando la concentración de ésta en el plasma de ratas diabéticas y normales ${ }^{(9-10)}$. Por otro lado, estudios clínicos preliminares demostraron que las raíces y hojas tienen actividad hipoglicemiante ${ }^{(11)}$.

La búsqueda de agentes naturales eficaces para controlar la diabetes y los antecedentes mencionados unidos a otros estudios sobre su composición nos motiva a buscar evidencias sobre el uso seguro de la infusión de hojas de Smallanthus sonchifolius (yacón), por lo que el objetivo del presente estudio fue evaluar el efecto hipoglicemiante de dicha infusión (extracto acuoso), administrada a pacientes con diabetes mellitus tipo 2 y a un grupo control aparentemente sano, a quienes se les determinaron los niveles de glucemia, hemoglobina glicosilada, fructosamina y el indice de masa corporal.

\section{MATERIALES Y MÉTODOS}

Las Hojas de Smallanthus sonchifolius (yacón) provienen de Huasahuasi, provincia de Tarma y fueron cosechadas, conservandosupedúnculo, al octavoynoveno mes de su ciclovital. Después de lavadas, se desecaron por aire forzado a $55^{\circ} \mathrm{C}$ por 48 horas. Se obtuvieron las hojas secas con $8 \%$ de humedad y se trituraron en un molino de cuchillas provisto de tamices con aberturas de 1,5-0,8 mm de diámetro. Cada filtrante de hojas de yacón se preparó pesando $1 \mathrm{~g}$ de este producto, que se envasó en bolsa de papel filtrante de celulosa, termosellable; este proceso se realizó en el Laboratorio de ENACO. La infusión se preparó por inmersión del filtrante en $250 \mathrm{~mL}$ de agua hirviendo, se tapó y dejó en reposo durante 5 minutos. Previamente se realizó una marcha fitoquímica para la investigación de los metabolitos secundarios utilizando reactivos de coloración y precipitación.

Participaron en el estudio, después de firmar su consentimiento informado de acuerdo a las normas internacionales vigentes, un total de 206 personas con edades comprendidas entre 30 a 70 años, de ambos sexos y domiciliadas en la ciudad de Lima:

105 pacientes con diagnóstico de diabetes mellitus tipo 2, que recibían como terapia glibenclamida, fueron divididos en: Grupo A1: 46 pacientes que tenían controlada la enfermedad, y Grupo A2: 59 pacientes que presentaban inadecuado control metabólico, demostrado por glucosa sérica en ayunas $\geq$ a $140 \mathrm{mg} /$ $\mathrm{dL}$, hemoglobina glicosilada $\geq \mathrm{a} 8, \mathrm{o} \%$ y fructosamina $\geq$ a $2,7 \mathrm{mmol} / \mathrm{L}$. Estos pacientes acuden a los siguientes lugares: Servicio Académico Asistencial de Análisis Clínicos (SAAAC), Gabinete de Atención Farmacéutica (GAF) de la Facultad de Farmacia y Bioquímica, Club de diabéticos tipo 2 del Hospital Cayetano Heredia y Centro de Salud (CERIT) Raúl Patrucco Puig.

101 personas aparentemente sanas sirvieron de Grupo Control, que fue dividido en: 6o sujetos normales (Grupo $\mathrm{B} 1$ ) y 41 sujetos sin diabetes mellitus tipo 2 con mal control metabólico (Grupo B2).

Los grupos A2 y $\mathrm{B} 2$ se sometieron a una ingesta diaria de infusión de $1 \mathrm{~g}$ de yacón tres veces al día, media hora antes de cada comida, durante 90 días. El grupo A2 continuó su tratamiento con glibenclamida.

A todos los grupos se les determinó el Índice de Masa Corporal y se les tomó muestras de sangre en ayunas para cuantificar las concentraciones de glucosa, hemoglobina glicosiladay fructosamina en las muestras basales y a los 30 y 90 días de la administración de la infusión de yacón.

Para el análisis estadístico de los resultados, se utilizó el programa Statistical Package for the Social Sciences (SPSS) versión 15.o. Los resultados fueron evaluados con 
estadística descriptiva. Se presentan los valores promedio \pm DS de los parámetros mencionados: basales, a los 30 y 90 días de tratamiento. Se consideraron significativas las diferencias entre medias cuyo valor de $p$ fuera $<0,05$.

\section{RESULTADOS}

Los resultados del análisis de las hojas secas fueron: humedad 7,7; proteína 15,4; grasas 12,5; cenizas 7,3; carbohidratos 48,4 y fibra 26,7 (g/10o g).

Los ensayos fitoquímicos preliminares, realizados en las hojas de yacón recolectadas, indicaron la presencia de compuestos de tipo triterpenos y esteroides, flavonoides, saponinas, fenoles, taninos, aminoácidos libres, ácidos grasos, sesquiterpenolactonas, así como trazas de azúcares similares a los reportados por otros autores ${ }^{(6,7)}$. No se detectó la presencia de antraquinonas, antocianinas, glucósidos cianogenéticos y alcaloides.

En el análisis de la infusión preparada por inmersión de $1 \mathrm{~g}$ de hojas de yacón en $250 \mathrm{~mL}$ de agua hirviendo, se encontró: Ca 2,29; Mg 1,62; Na 1,32; K 12,4; P o,38 (mg/100 $\mathrm{mL})$; y Cu 2,9; Fe 11,85; Mn 11,5; Zn 11 ( $\mu \mathrm{g} / 100 \mathrm{~mL})$.

En la tabla 1 se presentan los valores de glucosa, hemoglobina glicosilada y fructosamina, antes y después de 30 y 90 días de la administración de la infusión de hojas de yacón a los pacientes diabéticos y al grupo control. Estos resultados demuestran, en pacientes con diabetes mellitus tipo 2 no controlados, disminuciones en los niveles de los siguientes parámetros $(\mathrm{mg} / \mathrm{dL})$ : 224,27 a 128,51; hemoglobina glicosilada: de 11,13 a 8,87 (21\% de efectividad) y fructosamina sérica: 4,44 a 2,94 (33\% de efectividad), lo que corrobora la actividad normoglicemiante del extracto de hojas secas de yacón, con descenso gradual de la glucosa plasmática conforme avanza el tiempo de tratamiento, siendo las diferencias significativas con respecto al grupo control $(p<0,05)$. Los Îndices de Masa Corporal para diabéticos y controles inicialmente fueron de: $29,63 \pm 5,87$ y $27,44 \pm 2,36$ $(\mathrm{Kg} / \mathrm{m} 2)$, respectivamente. En 90 días los diabéticos perdieron peso con respecto al inicio del tratamiento,

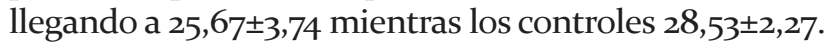

\section{DISCUSIÓN}

Nuestros resultados corroboran los de Inoue y col. ${ }^{(9)}$, quienes observaron que la infusión de hojas de yacón, administrada a ratas diabéticas por vía oral durante 30 días, redujo la glicemia en un $27 \%$. Según estos investigadores, la acción inhibitoria de esta preparación sobre la hiperglucemia inducida por estreptozotocina, puede atribuirse al hecho de que esta planta provoca un aumento en la concentración de insulina plasmática. Aybar ${ }^{(10)}$ demostró que la infusión de hojas de yacón, administrada a ratas diabéticas durante 30 días, tenía efecto hipoglucemiante y sugiere que ésta actúa mejorando la concentración de insulina en la sangre. Volpato ${ }^{(19)}$ administró extracto acuoso de las hojas de yacón a ratas diabéticas, demostrando que el extracto reduce los niveles de glucosa en sangre y reportó además que no altera los niveles de glucosa sanguínea en animales con glicemia normal. Mayta (11), estudiando "la reducción de la respuesta glicémica posprandial post-ingesta de raíz fresca de yacón en sujetos sanos", encontró que el consumo agudo de 300 g de raíz fresca, entera, de Smallanthus sonchifolius reduce la respuesta glicémica postprandial y evita el pico de glucosa durante el test de tolerancia oral a la glucosa, primero con una toma control y otra con la ingesta de raíz fresca de yacón. Da Silva ${ }^{(12)}$ evaluó la "respuesta de la glucosa en mujeres sanas después de la toma de yacón equivalentes a 50 g de carbohidratos, tomando como referencia el pan blanco durante el consumo de sus 
alimentos individualmente influenciando directamente en el control glucémico de las voluntarias". Bobadilla (13) observó disminución de glucosa en los pacientes diabéticos después de la ingesta de té de yacón, con un promedio de $51 \mathrm{mg} / \mathrm{dL}$ en las primeras tres semanas de estudio. Otros autores reportan que los valores de glicemia postprandial, durante 20 días de estudio, bajaron a menos de $180 \mathrm{mg} / \mathrm{dL}$. Tasayco ${ }^{(14)}$ demuestran que el extracto hidroalcohólico al 10\% p/v de las hojas de Smallanthus sonchifolius (yacón) tienen actividad hipoglucemiante en ratas con diabetes mellitus tipo 2.

En nuestroestudiosedemuestraque, al incluirinfusión de hojas de yacón en la dieta de: pacientes con diabetes mellitus tipo 2, y en la de sujetos sin diabetes mellitus tipo 2 con mal control metabólico, los niveles de glucosa se redujeron significativamente en 43 y $15 \%$, respectivamente.

Desde el punto de vista químico, la habilidad especial de Asteraceae de producir y acumular sesquiterpenolactonas debe ser resaltada. En nuestro caso utilizamos hojas secas preparadas para infusión , las cuales no contienen FOS (fructooligosacáridos) y presentan escasa cantidad de inulina. Los estudios del perfil fitoquímico de las hojas de yacón sugieren la presencia de flavonoides, melampolidos, triterpenos, esteroides, sesquiterpenos $(6,7,15,17)$. Es muy probable que los flavonoides sean responsables de la actividad normoglicemiante, pues se ha demostrado que participan en las etapas iniciales de acción de la insulina en el hígado y músculos de ratas in vivo, y uno de sus mecanismos de acción es ligarse a los receptores de insulina y potencializar la actividad de la enzima tirosinaquinasa de de dichos receptores. Esta enzima es fundamental para los efectos biológicos finales de la insulina, entre ellos reducir la glucemia ${ }^{(16)}$. Los flavonoides son apreciados por su amplia actividad farmacológica, pueden unirse a polímeros biológicos como: enzimas, transportadores de hormonas y ADN; quelar iones metálicos transitorios, tales como hierro, cobre y zinc; catalizar el transporte de electrones y depurar radicales libres. Debido a este hecho se han descrito efectos protectores en patologías como diabetes mellitus, cáncer, cardiopatías, infecciones víricas, úlceras estomacal y duodenal e inflamaciones.

Nuestros resultados demuestran que la infusión de las hojas de Smallantus sonchifolius (yacón) tiene actividad normoglicemiante ${ }^{(14)}$ en pacientes con diabetes mellitus tipo 2, pero no se ha establecido con claridad qué componente activo sería responsable de dicha actividad, ni el mecanismo exacto de acción, esto sólo se podrá explicar cuando los principios activos se encuentren aislados y se analicen en relación a esta actividad.
Además observamos que, el extracto de yacón, no produjo toxicidad observable en los animales de experimentación al aplicarse el ensayo descrito por las guías de la OECD TG 423, por lo que el producto podría considerarse prácticamente inócuo para los humanos cuando se administra de forma aguda, según la Clasificación de la Unión Europea.

Por otro lado, es ampliamente aceptado que la diabetes mellitus incrementa la producción de radicales libres derivados del oxígeno, tales como: anión superóxido $\left(\mathrm{O}_{2}\right)$, peróxido de hidrógeno $\left(\mathrm{H}_{2} \mathrm{O}_{2}\right)$ y radical hidroxilo $\left(\mathrm{OH}^{-}\right)$, superando los mecanismos de protección del organismo contra ellos. Bajo estas circunstancias pueden establecerse condiciones patológicas conocidas como estrés oxidante, las cuales pueden ser de tipo oxidante o nitrosante, dependiendo de las reacciones que predominen. En contraposición, los flavonoides desempeñan un papel esencial en la protección frente a los fenómenos de daño oxidativo, sus propiedades antirradicales libres se dirigen fundamentalmente hacia los radicales hidróxido y superóxido, especies altamente reactivas. Creemos que el yacón protege de las complicaciones producidas por la diabetes, lo que se sustentaría con nuestros resultados de la concentración de polifenoles, taninos y flavonoides. La fracción rica en lactonas sesquiterpénicas, principalmente fluctuanina, enhydrina, sonchifolina, uvedalina, y quercetina, que se encuentran en las hojas del yacón, presentan actividad hipoglucemiante, como fue propuesto por otros autores (7). Los flavonoides presentes en las hojas de Smallanthus sonchifolius tienen actividad antioxidante, inmunológica y no muestran toxicidad a las dosis ensayada, esto permite proponer a esta especie vegetal como un producto natural beneficioso para ser consumido por la población diabética o con riesgo de alteraciones metabólicas. La quercetina y sus efectos metabólicos han sido estudiados ampliamente y existen trabajos que demuestran la capacidad de este flavonol para mejorar la sensibilidad a la insulina y el metabolismo glucídico.

Es posible varios mecanismos por los cuales la planta reduce la concentración de glucosa en sangre: mayor liberación de insulina a través de la estimulación de las células B pancreáticas, la resistencia a las hormonas que incrementan la tasa de liberación delaglucosa, aumentodel número y la sensibilidad de los receptores de la insulina, la disminución de la degradación del glucógeno, el aumento en la captación de glucosa por los tejidos y órganos, y la reducción de la absorción intestinal de glucosa.

En la actualidad existen estudios sobre un tipo de glicósido de flavona (derivados de flavonoide) que 
demostró actividad hipoglicemiante debido a que estaría ligado a los receptores proliferadores de peroxisomas o antagonistas de receptores de glucagón, inhibidor dipeptidil peptidasa IV y activador de los receptores de insulina. Existen tres subtipos de PPAR: PPAR- $\alpha$, PPAR- $\gamma$ y PPAR- $\delta$. El PPAR- $\alpha$ se encuentra en el hígado, músculo esquelético y riñones, mientras que el PPAR- $\delta$ está ampliamente expresado. El PPAR- $\gamma$ está relacionado con la regulación y maduración del adipocito, siendo el sitio de acción para los fármacos sensibilizadores a la acción de la insulina, como la troglitazona, pioglitazona y rosiglitazona, los que conllevan a la reducción de la glicemia. Este blanco molecular podría ser el sitio de acción donde posiblemente actuarían los flavonoides presentes en la infusión de las hojas del yacon porque se ha demostrado actividad hipoglicemiante con derivados de flavonoides, como la quercetina y la isoorientina. La dipeptidil peptidasa IV (DPP-IV) es una enzima que degrada a la hormona incretina. El principal y predominante tipo de incretina es el GLP-1, que estimula la secreción de insulina, suprime la liberación de glucagón, enlentece el vaciamiento gástrico, mejora la sensibilidad a la insulina, y reduce el consumo de alimentos; y los flavonoides estarían favoreciendo la presencia del inhibidor dipeptidil peptidasa IV.

Otros metabolitos secundarios que estarían coadyuvando con el efecto hipoglicemiante son las sesquiterpenolactonas, que inducen la secreción de insulina solo en concentraciones altas de glucosa, lo cual disminuye el riesgo de hipoglicemia; esto ha sido confirmado mediante estudios in vitro, al aislar los islotes pancreáticos y someterlos a la sesquiterpenolactona tipo enhidrina, que determinó secreción de insulina. Por ejemplo, las sesquiterpenolactonas se han detectado en los tricomas glandulares de Smallanthus sonchifolius, lo que contribuye a los estudios sobre esta especie. Además, entre otros componentes menores de las glándulas también se han reportado flavonoides. No debería descartarse la idea de que al menos parte de los compuestos responsables delaactividad hipoglucemiante estén presentes en las sustancias almacenadas en los tricomas y canales esquizógenos de los tejidos foliares.

Según algunos estudios, el disolvente utilizado en la preparación de extractos de plantas puede afectar cualitativa y cuantitativamente a los componentes químicos biológicamente activos extraídos, por lo tanto, la forma de preparación es muy importante para su actividad biológica. Cabe destacar que, el efecto del extracto en la glucemia de ratas se observó solamente después de 14 días de tratamiento, lo que indica que las sustancias activas requieren un período de tiempo para alcanzar concentraciones eficaces en el organismo, de modo que el yacón pueda mejorar la hiperglucemia en la prueba de tolerancia a la glucosa.

La intoleranciaa laglucosa es conocida comounodelos factores patogénicos de la diabetes mellitus tipo 2, junto con la resistencia a la insulina, por lo tanto es importante conocer que el yacón mejora la tolerancia a la glucosa y no afecta a la glucosa en sangre en ayunas. A partir de estos resultados, se sugiere que el yacón tiene efectos hipoglicemiantes sin disminuir la producción de glucosa hepática.

En el presente estudio, el tratamiento coadyuvante de los diabéticos con la infusión de hojas de yacón reduce significativamente el IMC y los niveles de glucosa y fructosamina. Se puede apreciar que hay diferencia significativa entre el efecto normoglicémico, de la infusión de las hojas de $S$. sonchifolius, en pacientes diabéticos+tratamiento y los que continuaron sólo con el tratamiento farmacológico con glibenclamida. La reducción del nivel deglicemia evidenciada en la presente investigaciónseexplicaríaporlapresenciadecompuestos fenólicos, flavonoides y sesquiterpenolactonas en el yacón, o también por la presencia de otros metabolitos secundarios como las sesquiterpenolactonas (enidrina, uvedalina, sonchifolina, fluctuanina), que se encuentran y acumulan en los tricomas glandulares de las hojas o apéndices de anteras, relacionados a la defensa de la planta contra los depredadores, como lo afirma Schorr, et al ${ }^{(15)}$. La detección de estos componentes químicos por HPLC permitirá la identificación de varios metabolitos por medio de la distribución de los picos utilizando indirectamente compuestos de referencia auténticos, por lo que sería recomendable el desarrollo de nuevos estudios bioquímicos y farmacológicos a fin de aclarar la naturaleza de los metabolitos responsables y el exacto mecanismo de acción de estos compuestos.

\section{CONCLUSIONES}

Del análisis de los resultados obtenidos al evaluar el efecto de la ingesta de infusión ó extracto acuoso de las hojas de Smallanthus sonchifolius (yacón) en pacientes con diabetes mellitus tipo 2, podemos concluir que:

1. Los cambios se obtuvieron con la infusión preparada con hojas secas de Smallanthus sonchifollius (yacón) a la dosis de $3 \mathrm{~g}$ diarios, administrada por el lapso de 3 meses.

2. Reduce significativamente la glicemia a los 60 y 90 días en pacientes con diabetes mellitus tipo 2 con terapia de glibenclamida + tratamiento coadyuvante de infusión de hojas de Smallanthus sonchifolius, siendo mayor a los 90 días $(224,57-128,51 \mathrm{mg} / \mathrm{dL})$ con $43 \%$ de efectividad. 
3. Los pacientes con diabetes mellitus tipo 2, compensados con terapia de glibenclamida + tratamiento coadyuvante de infusión de hojas de Smallanthus sonchifolius, mostraron mayor reducción del nivel de glicemia, que los que sólo recibieron glibenclamida.

4. Disminuye los IMC, los niveles de glucosa,

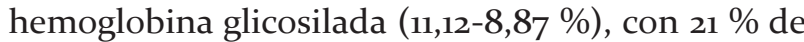
efectividad y fructosamina (4,44 a 2,94 $\mathrm{mmol} / \mathrm{L})$, con efectividad de $34 \%$.

5. El Índice de Masa Corporal $\left(\mathrm{Kg} / \mathrm{m}^{2}\right)$ para Diabéticos+medicamento+infusión de Smallanthus sonchifolius y Diabéticos+medicamento fueron de

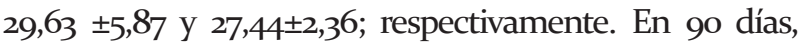
los primeros perdieron peso con respecto al inicio del

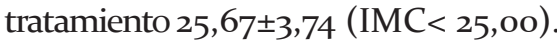

\section{REFERENCIAS BIBLIOGRÁFICAS}

1. Guías ALAD de diagnóstico control y tratamiento de la diabetes mellitus tipo 2. Revista de la Asociación Latinoamericana de Diabetes. Organización Panamericana de la Salud. Washington D.C., 2008.

2. OMS. Estrategia de la OMS sobre medicina tradicional 20022005. Organización Mundial de la Salud. Ginebra, 2002. [En línea] Acceso 10 abril 2008. Disponible en: http://whqlibdoc. who.int/hq/2002/WHO_EDM_TRM_2002.1_spa.pdf

3. National Research Council. Lost crops of the Incas: Little known plants of the Andes with promise for worldwide cultivation. [En línea] Acceso 15 de marzo 2006. Disponible en: http://www.nap.edu/openbook/030904264X/html/115.html

4. Barata L. Empirismo e ciência: fonte de novos fitomedicamentos. Cienc Cult 2005; 57(4). [En línea] Acceso 17 abril 2008. Disponible en: http://cienciaecultura.bvs.br/scielo. php?pid=s00o9-67252005000400002\&script=sci_arttext

5. López M. Plantas medicinales con actividad hipoglucemiante. Características, administración y efectos adversos. OFFARM 2006; 25(5): 82-8.

6. Kamimura GKF. Isolamento, purificação e caracterização da peroxidase de yacon (Smallanthus sonchifollius). 100p. Dissertação Mestrado em Alimentos e Nutrição - Faculdade de Ciências Farmacêuticas, Universidade Estadual Paulista, Araraquara, 2006. [En línea] Acceso 14 julio 2007. Disponíble en: http://www.biblioteca.unesp. br/bibliotecadigi tal/document/?did=4041

7. De Abreu J. Estudos químico e bioquímico do yacón (Smallanthus sonchifolius) in natura e processado e influência do seu consumo sobre níveis glicêmicos e lipídeos fecais de ratos. Para obtenção do Título de "Mestre" Ciência dos Alimentos. Universidade Federal de Lavras. Minas Gerais. 2008.
8. Valentova K, et al. Antioxidant activity of extracts from the leaves of Smallanthus sonchifolius. Eur J Nutr 2003; 42(1): 61-6.

9. Inoue A, Tamogami S, Kato H, Nakazato Y, Akiyama M, Kodama O, Akatsuka T, et al. Antifugal melampolides from leaf extracts of Smallanthus sonchifolius. Phytochemistry. 1995: 39 (4): 845-8.

10. Aybar J. et al. Hypoglycemic effect of the water extract of Smallanthus sonchifolius (yacon) leaves in normal and diabetic rats. J Ethnopharmacol 2001; 74(2): 125-32.

11. Mayta P, Payano J, Pelaez J y col. Reducción de la respuesta glicémica postprandial post-ingesta de raíz fresca de yacón en sujetos sanos. CIMEL 2004; 9(1): 7-11.

12. Da Silva A, Haas P, Beber R, Batista S, Antón A. Avaliação da Resposta Glicêmica Em Mulheres Saudáveis Após A Ingestão de Yacon (Smallantus Sonchifollius) In Natura, Cultivadas No Estado de Santa Catarina - Brasil. Alim. Nutr. Araraquara abr/jun.2006; 17 (2): 137-142.

13. Bobadilla M, Salazar V. Evaluación sobre el efecto de la inulina en la glucosa de pacientes con diabetes mellitus tipo 2. Cali, Colombia. 2007. [En línea] Acceso 25 abril 2008. Disponible en: http://colombia.acambiode.com/ empresa/cinafrix-ltda_189983

14. Tasayco Yataco, J. Actividad hipoglucemiante del extracto hidroalcohólico de las hojas de Smallanthus sonchifolius (yacón) en ratas con diabetes tipo 1 y 2 [Tesis para optar al Grado Académico de Magíster en Farmacología con mención en Farmacología Experimental] Fac. de Farmacia y Bioquímica - UNMSM. Lima, 2007.

15. Schorr K, et al. Quantitative determination of enhydrin in leaf rinse extracts and in glandular trichomes of Smallanthus sonchifolius (Asteraceae) by reversed-phase high-performance liquid chromatography. Phytochem Anal 2005; 16(3): 161-5.

16. Agullo G, GametPayrastre L, Manenti S, y col. Relationship between structure and inhibition of phosphatidylinositol 3-kinase: A comparison with tyrosime kinase and protein kinase C inhibition. Biochem Pharmacol 1997; 53: 16491657 .

17. Abrão de Oliveira, Luciana. Yacon (Smallanthus sonchifolius): Compostos fenólicos totais e efeitos sobre a glicemia e estresse oxidativo em ratos diabéticos. Universidade Estadual Paulista "Julio De Mesquita Filho" Faculdade de Ciências Farmacêuticas. Araraquara, 2010.

Manuscrito recibido el: 12/11/2012

Aceptado para su publicación el: 10/11/2012

\section{Correspondencia:}

Nombre: Gloria Gordillo Rocha

Dirección: Jr. Puno 1002- Cercado de Lima, Perú

e-mail: ggordillor@unmsm.edu.pe 